\title{
An Approach for Coverage Path Planning for UAVs
}

\author{
L. H. Nam ${ }^{\mathrm{a}}$, L. Huang ${ }^{\mathrm{a}}$, X. J. Li ${ }^{\mathrm{a}}$ and J. F. Xu ${ }^{\mathrm{b}}$ \\ ${ }^{a}$ School of Engineering. Computer and Mathematical Sciences \\ Auckland University of Technology, Auckland, New Zealand \\ namle@aut.ac.nz, lhuang@aut.ac.nz, xuli@aut.ac.nz \\ b State Key Laboratory of Digital Manufacturing Equipment and Technology \\ Huazhong University of Science and Technology, Wuhan, China \\ jfxu@hust.edu.cn \\ * Corresponding author
}

\begin{abstract}
In this paper, an offline flight planner that computes an efficient coverage trajectory for a quad-rotors UAV is presented. The planner consists of three steps: mission definition, automatic path planning and trajectory generation. The proposed planner, as a useful tool, allows an UAV operator to easily define and generate a coverage trajectory for any specific task. The resultant trajectory can be dispatched to a quad-rotors with trajectory tracking controller for the missions that require a complete area coverage.
\end{abstract} rotors.

Keywords-Coverage trajectory planning, Aerial robots, Quad-

\section{INTRODUCTION}

Image surveys on a large area are important in many areas including ecological research and precision agriculture. For example, unmanned aerial vehicles (UAVs) with on-board sensors like cameras are commonly used to carry out image surveys over a large farm field (Valente et al., 2012; Polo et.al, 2015). The generated maps are usually built by mosaicking a set of overlapped geo-referenced images recorded in the flight mission of the UAVs. They provide the comprehensive data about crop condition and other relevant parameters of the field. Waypoint GPS navigation are universally implemented in the survey (Link et al., 2013; Urbahs \& Jonaite, 2013). Waypoint GPS navigation allows a UAV to fly on its own to its flying destination or points pre-planned and configured into the UAV navigational program. Hence, the UAV is able to scan the full field by following a continuous and smooth trajectory and simultaneously avoid areas that are not subjects of interest.

The key components for the UAV's survey missions are the UAV platform and the corresponding flight planner. There are mainly three types of UAV platforms: fixed-wing planes, helicopter and quad-rotors. Fixed-wing planes have advantages in speed and flight height and are suitable for large area surveillance. However, due to their high flying speed, they requires high speed sensors for survey. In addition, it is difficult to keep a fixed wing plane at a position and to take it off or land it in small spaces. Helicopters are superior with regard to path planning and maneuverability. Nevertheless, they require extensive maintenance and are difficult to control. Quad-rotors have the similar advantages of helicopters: hovering capability and decoupling between orientation and position motions. However it has a simple structure consisting of four rotors on a cross-shaped frame which also carry the main-board, sensors and other supporting modules. The changes in attitude and elevation are obtained by controlling the speeds of the four motors. Quad-rotors are inexpensive with good maneuverability, but their small pay-load capacity limits the weight of onboard sensors and flight time. Hence, they are more suitable for the survey missions in a small scale. Due to their high maneuverability combining with the readily available autopilot program, quad-rotors are widely applied in surveillance mission nowadays.

In a survey mission, a key task of the flight planner is to generate the UAV's path to completely cover the area of interest efficiently, and it is tackled with coverage path planning (CPP) technique. CPP is a sub-topic of motion planning for robots, which deals with methods to determine a path that ensures complete coverage while avoiding obstacles.

The CPP can accomplish verifiable coverage guarantee by using cell decomposition. A cell decomposition breaks down the target region into cells that can be covered easily. The complete coverage is accomplished by ensuring that the robot visits each cell once. The workplace is generally mapped in exact cellular decomposition or approximate cellular decomposition.

The exact cellular decomposition method develops from the trapezoidal decomposition, in which each cell's shape is a trapezoid. Simple back-and-forth motions can be used to cover each cell. The drawback of this methods is that the UAV's camera field of view (FOV) is ignored. It can lead to high overlapping in coverage, thus reduces the efficiency of the UAV survey. To solve this problem, Li et al. (2011) proposed an enhanced exact cellular decomposition method to plan the coverage path of UAVs in a polygon area. An algorithm to calculate the widths of convex polygons is developed and a path of the least number of turns for an UAV based on the width of the convex polygons is developed. Better results in path planning were obtained by avoiding unnecessary back and forth motions. However, the camera's FOV is still neglected.

An approximate cellular decomposition CPP for UAVs in agriculture application was introduced in (Valente et al. 2012). 
An approximate cellular decomposition is a grid-based representation of the working area. Each cell has the same size and shape, and the union of the cells approximates the target region. The size of the cell is determined by the camera's FOV. When the robot visits each cell's center in the decomposition, the coverage is complete. The path of the robot can be determined through spanning trees, neural networks, genetic algorithms, and general heuristic search algorithms. The approximate cellular decomposition combined with wave front algorithm with gradient ascent can be used to determine the coverage path with minimal number of turns (Valente et al. 2012). In addition, the so called Pedestrian Pocket Algorithm (PPA) based on the backtracking method can be used to find near optimal path and Quad Tree Algorithm can be used to reduce the computation time. To make the UAV return to the base station before it is out of power, the shortest return path for the UAV is computed using wave front algorithm with the steepest gradient descent method. However, in this case, the criteria of minimum number of turns is no longer relevant to the path planning as it may lead to longer travelling distance. At the implementation stage, the flight planner is only required to input a set of waypoints to the UAV's built-in auto pilot to generate a smooth trajectory for the UAV to follow.

This paper is to present an approach of coverage path planning for a UAV in a survey mission over a given site using approximate cellular decomposition and wave front algorithm. A new optimal criteria based on both route length and number of turns is utilized for the path planning. The rest of this paper is organized as follows. In Section II, the main algorithms for the path planning are described. Section III presents the experimental results. Conclusions are given in Section IV.

\section{Algorithmas}

\section{A. Workplace Sampling}

The workspace is segmented through an approximate cellular decomposition. In this method, the working place is divided into rectangles, and a point is placed in the center of each rectangle. The UAV is assumed to fully cover the cell when it reaches that particular point. The size of rectangles is calculated from FOV of the camera and the overlap rates required in mosaicking process.

In the grid based decomposition scheme, workplace $\mathrm{A}$ is transformed in to a Grid graph $G_{<V, E>}$ where $V$ and $E$ are used to denote the vertices and the edges of the cells, respectively. Each vertex is the center of the sampling cell and each edge is the path connecting the adjacent cells (Fig 1).

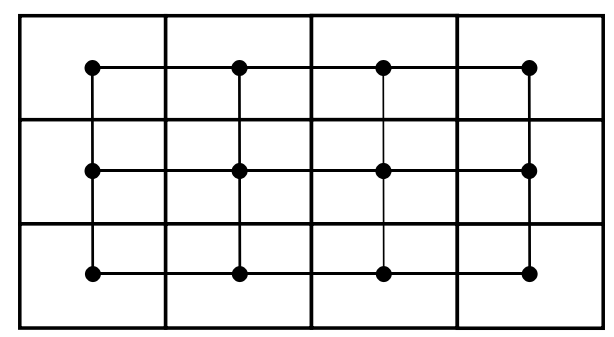

Fig. 1: A grid graph example
Camera's FOV is the area covered by UAV's camera when it is flying at altitude $\mathrm{h}$ (Fig 2a). The dimension of camera's FOV can be obtained by the following equations:

$$
\begin{aligned}
& W=2 h \times \tan \left(\frac{\alpha}{2}\right) \\
& L=2 h \times \tan \left(\frac{\beta}{2}\right)
\end{aligned}
$$

where
$W$ : width of FOV
$L$ : length of FOV
$h$ : flight altitude
$\alpha$ : vertical degree of camera
$\beta$ : horizontal degree of camera

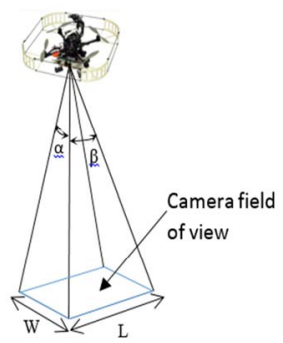

a)

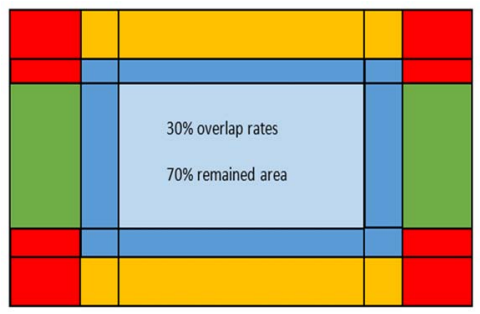

b)

Fig. 2: a) Camera FOV; b) Overlapping rate

Image mosaicking is the process of joining overlapping images together to form a larger image, hence enlarging a cameras' FOV. Stitching overlapping images requires the images to have an overlapping rated to overcome lens distortion and have enough detectable features (Fig 2b). The relationship between the areas of sampling cell and camera's FOV is:

$$
S_{\text {cell }}=(1-d) \times S_{F O V}
$$

where

$S_{\text {cell }}:$ area of sampling cell

$S_{F O V}=W \times F:$ area of camera's FOV

$d:$ overlap rate

\section{B. Wave front algorithm}

Consider the navigation function $\phi: G \rightarrow[1, \infty]$ which has the same feature as a potential function. For the goal cell, the navigation $\operatorname{cost} \phi\left(c_{G}\right)=1$ and the further the cell position to the goal cell the larger the navigation cost. The way point $u^{*}$, which 
is at the adjacent cell of the current way point $\mathrm{x}$, can be selected to reduce the potential as much as possible (gradient descending) or increase the potential as much as possible (gradient ascending).

$$
\begin{aligned}
& \text { Gradient descending } u^{*}=\operatorname{argmin}\{\phi(u)\}, u \in U(c) \\
& \text { Gradient ascending } u^{*}=\operatorname{argmax}\{\phi(u)\}, u \in U(c)
\end{aligned}
$$

where

\section{$c$ : current cell}

$U(c)$ : set of adjacent cells of c

$u^{*}$ : next cell in path planning

Gradient descending searching algorithm is commonly used in robot path planning to find the shortest path from the start cell to the goal cell. For the coverage planning, gradient ascending searching algorithm should be adopted where all the cells with higher value than goal cell is guaranteed to be visited before the robot reach the goal.

Navigation function can be established with wavefront propagation algorithm which is as follows:

1. Initialize $W_{0}=c_{G} ; \phi\left(c_{G}\right)=1$

2. Initialize $W_{i+1}=\emptyset$.

3. For every $c \in W_{i}$, assign $\phi(c)=i$ and insert all unexplored neighbors of $x$ into $W_{i+1}$

4. If $W_{i+1}=\varnothing$, then terminate; otherwise, let $\mathrm{i}=\mathrm{i}+1$ and go to step 2 .

In order to construct a navigation function, we must consider the type of cell connectivity based on the maneuverability of robot. There are two types of connectivity: the Von Neumann neighborhood (Fig. 3a) and Moore neighborhood (Fig. 3b). In a Von Neumann neighborhood, the aerial robot turning angle is limited to \pm 90 . In Moore neighborhood the robot will be able to turn $\pm 45, \pm 90$, or \pm 135 . For a quad-rotors UAV, it can rotate to any yaw angle value by changing the velocity of each motors. Therefore, the Moore neighborhood is chosen.

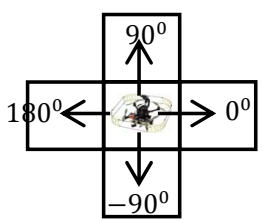

a)

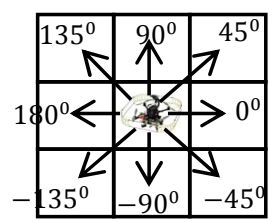

b)
Fig. 3: a) Von Neumann neighborhood b) Moore neighborhood

During the gradient tracking, the algorithm may find more than one neighbor with the same potential. The unexpanded nodes needs to be recorded and labeled as a state of bottle neck. The bottleneck is a state caused by the local minimal during the search. In this case, the current searching path can't completely cover the working area. A new search needs to be started from the unexpanded nodes. Hence, a backtracker needs to be implemented to record the unexpanded nodes. The result of this gradient ascending searching algorithm is a set of waypoints that will ensure complete coverage. Each way points contain information of $x, y$ and yaw angle $\varphi$.

As a result of path planning in the grid based environment, the survey path comprises of multiple straight segments with different angles as show in Fig 4. Therefore, it is assumed that the UAV will move with maximum velocity in straight line, stop for rotating at the end of one straight segment and continue to move in the next segments. The amount of time to complete the path can be calculated:

$$
T=\frac{S}{V}+\sum_{i=1}^{k} \frac{\varphi_{i}}{\omega}
$$

where
$T$ : completion time
$S$ : route length
$V$ : UAV movement speed
$k$ : number of turns
$\varphi_{i}$ : angle of the $i^{\text {th }}$ turn
$\omega$ : UAV rotation rate

An optimal path will be chosen to minimize the task completion time (Fig. 4).

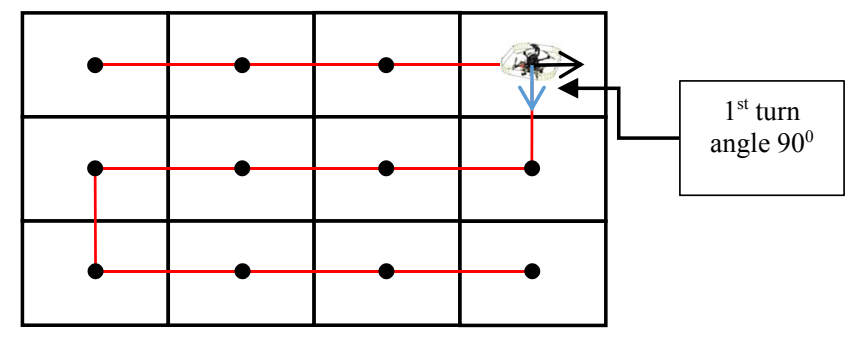

Fig. 4: Example of UAV coverage path

\section{Cubic spline}

A smooth trajectory can be generated from the solutions of path planning to improve the efficiency of the mission. With the trajectory information, UAV controller can manipulate the UAV navigation better so as to reduce the mission completion time.

For quad rotors, a single trajectory segment between two points in state space consists of independent polynomial trajectories for the variables $x, y$ and yaw angle $\varphi$. Cubic spline is a simple and effective method to generate a trajectory with $\mathrm{C}^{2}$ continuity. For the given points $\left(x_{i}, y_{i}, \varphi_{i}\right), i=0 \ldots n$, the algorithm to generate trajectory $S x(t)$ is described as below.

$$
\begin{aligned}
& S x(t) \\
& =\left\{\begin{array}{c}
S x_{0}(x)=a_{0} t^{3}+b_{0} t^{2}+c_{0} t+d_{0}, t_{0}<t<t_{1} \\
\vdots \\
S x_{n-1}(x)=a_{n-1} t^{3}+b_{n-1} t^{2}+c_{n-1} t+d_{n-1}, t_{n-1}<t<t_{n}
\end{array}\right.
\end{aligned}
$$


where

$n+1$ : total number of way points

$t_{0}=0$

$t_{n}=T$

$S x(t)$ must satisfy the following $\mathrm{C}^{2}$ continuity condition.

$$
S x(t) \in C^{2}\left[t_{0} ; t_{n}\right]:\left\{\begin{array}{l}
S x_{i-1}\left(t_{i}\right)=S x_{i}\left(t_{i}\right) \\
S x_{i-1}^{\prime}\left(t_{i}\right)=S x_{i}^{\prime}\left(t_{i}\right), i=1,2, \ldots, n-1 \\
S x_{i-1}^{\prime \prime}\left(t_{i}\right)=S x_{i}^{\prime \prime}\left(t_{i}\right)
\end{array}\right.
$$

The parameter value $t_{i}$ for the $(\mathrm{i}+1)^{\text {th }}$ point is chosen by Lee's centripetal scheme (Lee, 1989),

$$
\begin{gathered}
t_{0}=0 \\
t_{n}=T \\
\frac{t_{i}-t_{i-1}}{T}=\frac{\left|P_{i}-P_{i-1}\right|}{\sum_{i=1}^{n}\left|P_{i}-P_{i-1}\right|}, i=1, \ldots, n
\end{gathered}
$$

where $P_{i}=\left(x_{i}, y_{i}\right)$ is the $(\mathrm{i}+1)^{\text {th }}$ waypoint.

The requirements for a cubic spline gives $3(\mathrm{n}-1)$ equations. In addition, we require that $x\left(t_{i}\right)=x_{i}(\mathrm{i}=0, \cdots, n)$ which gives $n+1$ equations. This means we have $4 \mathrm{n}-2$ equations in total. We have $4 \mathrm{n}$ degrees of freedom $\left(a_{i}, b_{i}, c_{i}, d_{i}\right)_{i=0}^{n-1}$. Because the UAV starts from rest and stop when reaching end points, the last two equations are $S x_{0}^{\prime \prime}\left(t_{0}\right)=S_{n-1}^{\prime \prime}\left(t_{n}\right)=0$.

First, apply some pre-calculations

$$
\begin{gathered}
h_{i}=t_{i+1}-t_{i}, i=0, \ldots, n-1 \\
b_{i}=\frac{1}{h_{i}}\left(x_{i+1}-x_{i}\right), i=0, \ldots, n-1 \\
v_{i}=2\left(h_{i-1}+h_{i}\right), i=1, \ldots, n-1 \\
u_{i}=6\left(b_{i}-b_{i-1}\right), i=1, \ldots, n-1 \\
z_{0}=z_{n}=0
\end{gathered}
$$

Then, solve tri-diagonal system of $\left(z_{i}\right)_{i=1}^{n-1}$

$$
\left[\begin{array}{cccccc}
v_{1} & h_{1} & & & & \\
h_{1} & v_{2} & h_{2} & & & \\
& h_{2} & v_{3} & h_{3} & & \\
& & \ddots & \ddots & \ddots & \\
& & & \ddots & \ddots & h_{n-2} \\
& & & & h_{n-2} & v_{n-1}
\end{array}\right]\left[\begin{array}{c}
z_{1} \\
z_{2} \\
z_{3} \\
\vdots \\
z_{n-2} \\
z_{n-1}
\end{array}\right]=\left[\begin{array}{c}
u_{1} \\
u_{2} \\
u_{3} \\
\vdots \\
u_{n-2} \\
u_{n-1}
\end{array}\right]
$$

Substitute $\left(z_{i}\right)_{i=0}^{n}$ into following expressions to find the cubic spline

$$
\begin{aligned}
S x_{i}= & \frac{z_{i+1}}{6 h_{i}}\left(t-t_{i}\right)^{3}+\frac{z_{i}}{6 h_{i}}\left(t_{i+1}-t\right)^{3} \\
& +\left(\frac{x_{i+1}}{h_{i}}-\frac{z_{i+1} h_{i}}{6}\right)\left(t-t_{i}\right) \\
& +\left(\frac{x_{i}}{h_{i}}-\frac{z_{i} h_{i}}{6}\right)\left(t_{i+1}-t\right)
\end{aligned}
$$

Trajectory generation for variable $x$ is completed and the same method is applied to generate trajectories for $y$ and $\varphi$.

\section{Flight planner algorithm}

The flowchart of flight planner to generate UAV coverage trajectory is given in Fig. 5.

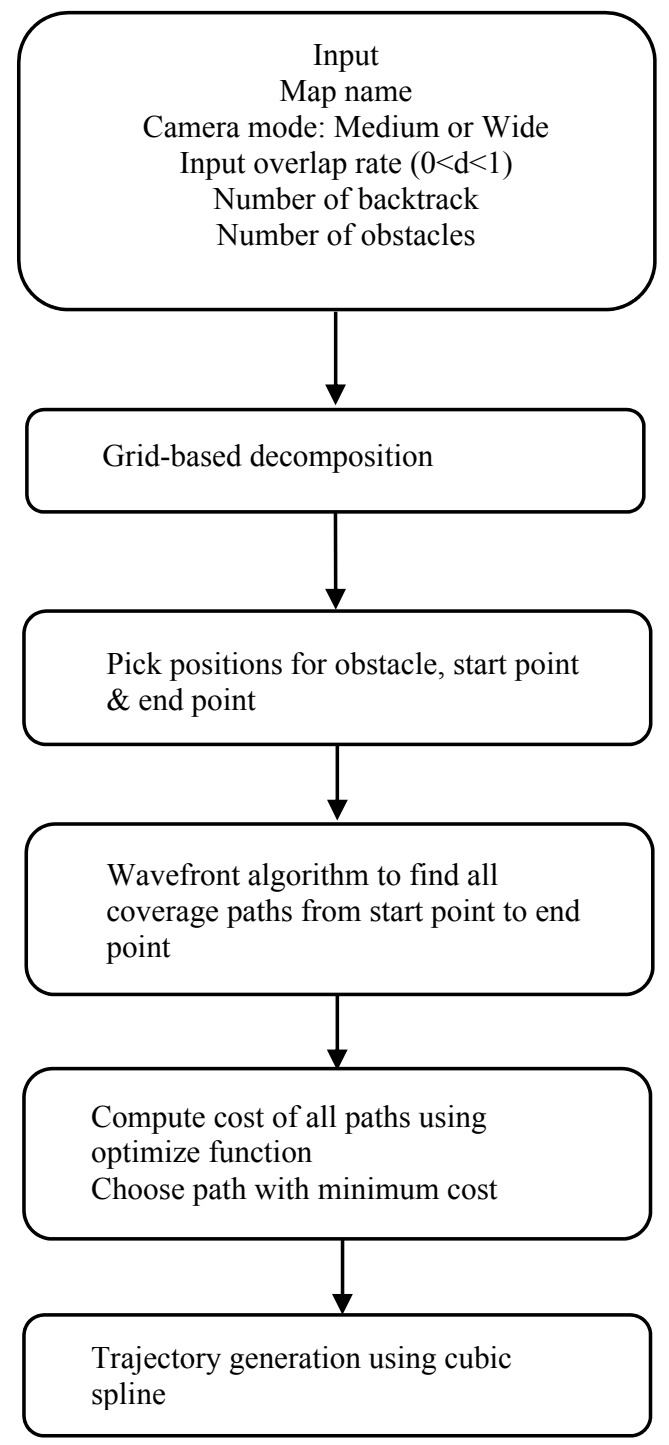

Fig. 5. Flowchart of the proposed flight planner

\section{RESULT}

The site of working area for this mission is Best Berries farm located at Coatesville, North Island, New Zealand. The initial map of the area is obtain from satellite image. The dimension of the workplace is calculated to be $137 \times$ $218 \mathrm{~m}^{2}$ from GPS coordinates of four corners A, B, C and D as shown in Fig. 6. 


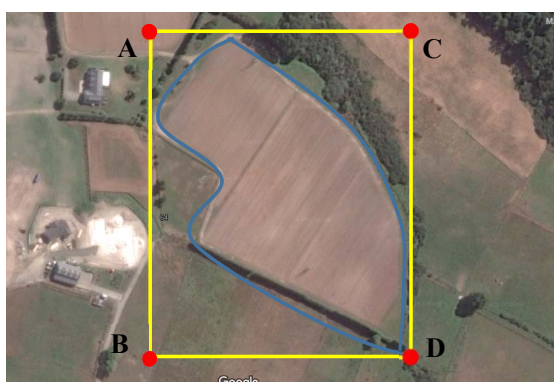

A: $-36.710724 \mathrm{~N}$,

$174.628808 \mathrm{~W}$

B: $-36.710726 \mathrm{~N}$

$174.630570 \mathrm{~W}$

$\mathrm{C}:-36.712546 \mathrm{~N}$

$174.628802 \mathrm{~W}$

D: $-36.712546 \mathrm{~N}$

$174.630573 \mathrm{~W}$

Fig. 6: Workplace identification

Table I. Workplace Parameters

\begin{tabular}{|l|l|l|}
\hline Workplace & Width & Length \\
\hline Size & $137 \mathrm{~m}$ & $218 \mathrm{~m}$ \\
\hline
\end{tabular}

Gopro Hero3 camera is the image sensor of the UAV. It has the following characteristics

Table II. Gopro Hero 3 Camera Parameters

\begin{tabular}{|c|c|c|}
\hline Image resolution & $11 \mathrm{MP}$ & $3840 \times 2880$ pixels \\
\hline Camera mode & Vertical degree & Horizontal degree \\
\hline $16 \times 9 \mathrm{M}$ & 55 & 94.4 \\
\hline $16 \times 9 \mathrm{~W}$ & 69.5 & 125.3 \\
\hline
\end{tabular}

The result of grid based decomposition based on two camera working mode M(medium) and W(wide) is presented in Fig 7.

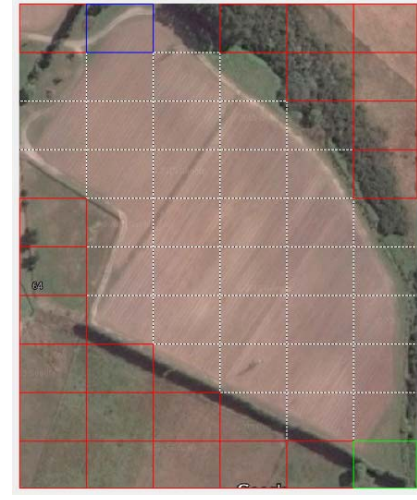

a)

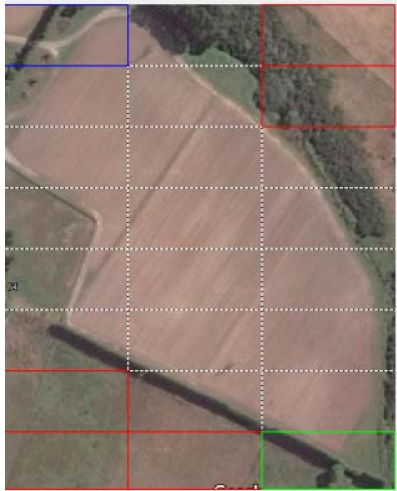

b)
Fig. 7: a) Grid-based decomposition with camera in Medium mode b) Grid-based decomposition with camera in Wide mode

Wavefront algorithm with ascending gradient searching and backtracker is implemented to determine the coverage path.

The solution is the path that minimize the completion time:

$$
T=\frac{S}{V}+\sum_{i=1}^{k} \frac{\varphi_{i}}{\omega}
$$

The UAV model using in this experiment is Pelican model of Asctec. Its maximum cruising speed is $16 \mathrm{~m} / \mathrm{s}$ and rotation speed is $60 \mathrm{deg} / \mathrm{second}$. The optimal path is presented in Fig 8.

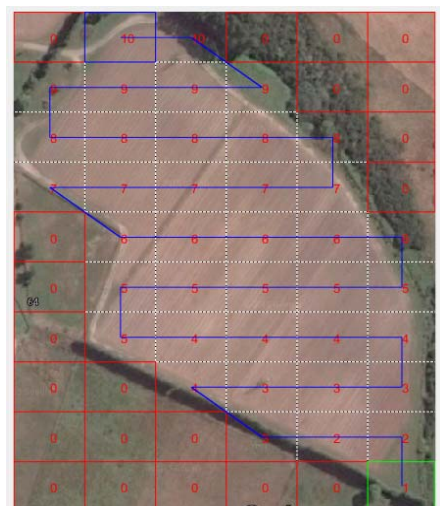

Fig. 8: Optimal path generated by wavefront algorithm

The cubic interpolation algorithm is applied to generate a smooth trajectory as shown in Fig. 9.

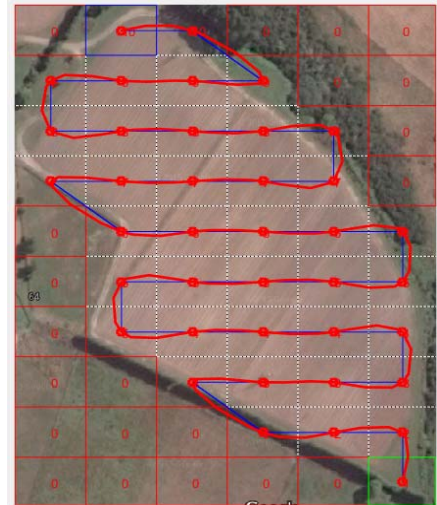

Fig. 9: Coverage trajectory generated by Cubic interpolation

The trajectory generated by cubic interpolation is smoother. than the flight path of UAV (Fig. 10) based on the GPS tracking navigation system developed by Valente et.al (2012).

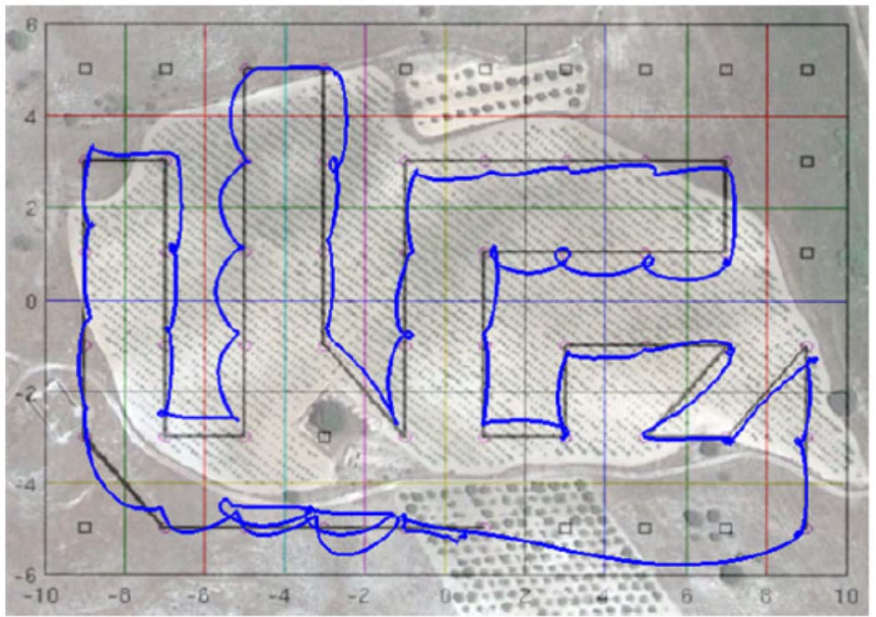

Fig. 10: UAV real flight trajectory using GPS tracking navigation (Valente et.al. 2012) 


\section{CONCLUSION}

In this paper, a complete flight planner that generates a coverage trajectory with minimum completion time for quadrotors UAV has been developed. Experimental results show that the trajectory provides a better navigation for UAV than GPS coordinates provided from the existing CPP algorithms.

\section{ACKNOWLEGDEMENT}

The work reported in the paper is jointly supported by the Auckland University of Technology and State Key Laboratory of Digital Manufacturing Equipment and Technology; Project No: DMETKF2015005.

\section{REFERENCES}

[1] J. Valente, D. Sanz, J. Del Cerro, A. Barrientos and M. A. de Frutos, “ Near-optimal coverage trajectories for image mosaicing using a mini quad-rotor over irregular-shaped fields," in Precision agriculture, Vol 14(1), pp. 115-132, 2013.

[2] J. Polo, G. Hornero, C. Duijneveld, A. Garcia and O. Casas, "Design of a low cost wireless sensor network with UAV mobile node for agricultural applications," in Computers and Electronics in Agriculture, Vol. 119, pp. 19-32., 2015.

[3] J. Link, D. Senner and W. Claupein, "Developing and evaluating an aerial sensor platform (ASP) to collect multispectral data for deriving management decisions in precision farming," in Computers and electronics in agriculture, Vol. 94, pp. 20-28. 2013.

[4] A. Urbahs and I. Jonaite, "Features of the use of unmanned aerial vehicles for agriculture applications," in Aviations, Vol. 17(4), pp. 170-175, 2013.

[5] Y. Li, H. Chen, M. J. Er and X. Wang, "Coverage path planning for UAVs based on enhanced exact cellular decomposition method," in Mechatronics, Vol. 21(5), pp. 876-885, 2011.

[6] E.T.Y. Lee, "Choosing nodes in parametric curve interpolation", in Computer Aided Design, Vol 21(6), pp.363-370, 1989. 